UDC 621.548

\title{
EFFECTS OF ELECTROHYDROIMPULSE DISCHARGES ON THE CRUSHING OF THE NATURAL MINERAL QUARTZ
}

\author{
Bulkairova G.A. ${ }^{1}$, Stoev M. ${ }^{2}$, Khassenov A.K. ${ }^{1}$, Karabekova D.Zh. ${ }^{1}$, Nurgaliyeva Zh.G. ${ }^{1}$, \\ Kipshakov M.S. ${ }^{1}$
}

1E.A. Buketov Karaganda State University, Karaganda, Kazakhstan, gulden2111@@mail.ru
22 Neofit Rila University, South-West, Blagoevgrad, Bulgaria

\begin{abstract}
Electro-hydro-impulse (high-current discharge in water) also belongs to the technologies using strong impulse currents. With a powerful pulsed electric discharge between the electrodes placed in the liquid, an electro-hydraulic effect occurs. To study the influence of electro-hydro-pulse shock waves were designed and assembled the experimental setup. The dependences of the degree of grinding of natural mineral quartz on the electro-technical parameters of the discharges are given. The optimal conditions for the most intensive grinding of natural mineral quartz using electro-hydro-pulse treatment are determined.
\end{abstract}

Keywords: natural mineral quartz, electro-hydraulic effect, underwater electric discharge, degree of grinding.

\section{Introduction}

The growing consumption of fuel, ferrous, non-ferrous and rare metals, non-metallic materials, which are the basis for the development of modern industry, requires an increase in the volume of extraction and enrichment of minerals. Together with non-metal ore, construction materials and mining and chemical raw materials, the volume of mining and processing of minerals in the world is several billion tons per year.

Processing of most of the mined rock mass means crushing and grinding it as a preparatory process for direct enrichment. These processes are very expensive operations and reach $50 \%$, and in some cases $70 \%$ of all costs in the processing plants. Of great importance for subsequent technological operations is the quality of crushing and grinding, which involves obtaining a product of a given size without re-grinding with maximum release of mineral grains from the waste rock with minimal damage. The requirements of increasing the number of processed rocks and ores while improving the quality indicators of processing (increasing the degree of extraction) pose very urgent problems aimed at streamlining and reducing the cost of crushing and grinding processes.

Rocks are mostly materials that break down brittle under normal conditions. In this regard, when conducting studies of physical processes occurring during the destruction, the most suitable model materials are brittle crystals. Quartz is a part of many igneous and metamorphic rocks: granite, porphyry, gneiss. The total content of quartz in igneous rocks is approximately $12 \%$. The hardness of quartz is 7 units on the Mohs scale. Melting point is $1710^{\circ} \mathrm{C}$.

The Republic of Kazakhstan has large reserves of quartz-containing raw materials, but most of it is used in the production of simple building materials, and only a small part-high-tech industries.

Quartz-one of the most common minerals, which is more or less pure silica $\mathrm{SiO}_{2}(46.7 \%-\mathrm{Si}$; $53.3 \%-\mathrm{O}_{2}$ ). Practically, quartz crystals rarely approach this composition, as they usually contain various impurities: bubbles of gases and liquids, sometimes ingrown mica leaves. Quartz is one of the most common minerals, which is more or less pure silicic acid. Practically, quartz crystals rarely approach this composition, as they usually contain various impurities: bubbles of gases and liquids, sometimes ingrown mica leaves. 
This article presents the results of the study of the influence of electro-hydro-impulsive shock waves on the crushing and grinding of the natural mineral quartz. Electro-hydro-impulse (highcurrent discharge in water) also belongs to the technologies using strong pulse currents. With a powerful pulsed electric discharge between the electrodes placed in the liquid, there is an electrohydraulic Yutkin effect, which consists in the fact that the rapid release of energy in the discharge channel increases the pressure in it, and its further expansion leads to a shock wave and fluid flows [1]. The shock wave is a density jump in the liquid that propagates from the discharge channel at a speed exceeding the sound (in water more than $1500 \mathrm{~m} / \mathrm{s}$ ). Pressure at the shock wave front in the liquid can reach tens of kilobars. The impact of this pressure on the treated object can cause structural restructuring of the object material (crushing brittle materials, deformation, hardening of the surface, etc.). Fluid flows propagating at a speed of $10^{2} \div 10^{3} \mathrm{~m} / \mathrm{s}$ transmit kinetic energy to the object being processed, causing, like a shock wave, its mechanical changes [2]. Mechanical manifestations of pulsed discharge in a liquid are called electro-hydraulic effect, and installations using this effect are called electro-hydraulic. As a working medium in such installations, as a rule, process water, kerosene, oils are used [3,4].

\section{Experimental technique}

To study the effect of electro-hydro-impulsive shock waves on the crushing and grinding of the quartz mineral, an experimental unit was developed and assembled in the Laboratory of Electrohydro-dynamics of the Engineering Thermophysics Department named after professor Zh.S. Akylbayev of the E.A. Buketov Karaganda State University.

Electro-hydraulic devices for crushing unlike mechanical crushers do not have moving parts, are made of conventional structural steel, and their body practically does not wear out during operation. During operation, these devices do not form dust, occupy a relatively small production area and allow them to combine the processes of crushing, mixing and flotation of materials [4].

The installation consists of the following units: pulse voltage generator, capacitor and protection system, control panel and electrode system. Figure 1 shows the working part of the installation, which consists of a cylindrical body 1 , working electrodes 2 , cover 3 , caprolan washer 4 and caprolan sleeve 5 .

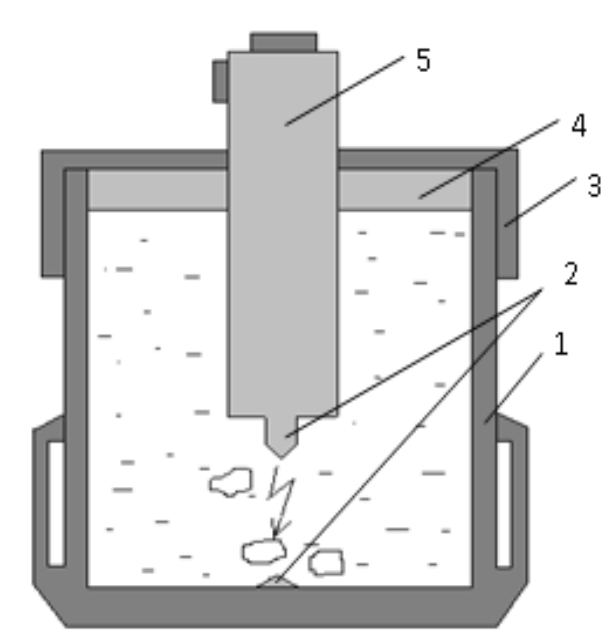

Fig.1. Working part of the experimental plant for crushing quartz mineral.

In the working part of the experimental installation, a linear system of electrodes is installed. The positive electrode is located vertically, and the negative electrode is the bottom of the cylindrical metal chamber. To obtain high pressures at the shock wave front, crushing and grinding solid fractions, an electric discharge is carried out in an aqueous solution of the mineral quartz [5]. 
The rapid release of energy generates a strong mechanical action applied to the macroscopic volumes of the medium and individual surfaces that have fallen into the discharge zone, at the same time, the rate of energy release is much faster than the rate of transmission of its perturbation to the environment. After the beginning of the breakdown occurs for some time, the so-called stage of formation of the discharge, which is characterized by a certain increase in current with a simultaneous decline in voltage and culminating in the formation of a highly conductive electric channel. The energy accumulated by the capacitor Bank is introduced into the formed zone. Under the action of high pressure, the discharge channel expands, the macroscopic volumes of the medium receive relatively high velocities, which are directed in all directions along radii originating approximately from the Central part of the discharge gap. Under the influence of high pressure, the liquid is compressed, the area of this compression ends with a moving shock wave front, where the pressure and all other parameters of the liquid medium are changed by a pressure jump. The energy of the heated plasma is transferred directly to the environment. Electrohydraulic crushing devices unlike mechanical crushers do not have moving parts, are made of conventional structural steel, and their body practically does not wear out during operation. During operation, these devices do not form dust, occupy a relatively small production area and allow them to combine the processes of crushing, mixing and flotation of materials. The liquid, having received acceleration from the discharge channel expanding at high speed, moves from it in all directions, forming in the place where the discharge was a significant cavity in volume (cavitation) causing the first (main) hydraulic shock. The development of a spark discharge in time occurs by successive «germination» of streamers in the inter-electrode interval. A growing streamer, as a rule, consists not of one, but of many channels with numerous branches from them.

Under the influence of electric pulse action in the treated medium there are hydrodynamic fluid flows and acoustic wave, and as a result of local pressure reduction in the liquid cavitation occurs. In this case, the cavitation bubble, moving with the flow of liquid to the area with a higher pressure, closes and emits a shock wave. After the collapse of the bubbles, micro-shocks of cumulative jets will form. The mixture, having received acceleration from the discharge channel expanding at high speed, moves from it in all directions. At the beginning of the process, the discharge channel increases with the maximum speed, at the end of the current flow, the cavity of the discharge channel continues to expand due to the inertia of the medium, reaches the largest size and then begins to shrink. The temperature and pressure in it during the expansion of the cavity fall, and increase during compression, i.e., there are damping pulsations of the cavity [6]. After a series of discharges, the natural mineral quartz is destroyed, the processing products are extracted and the cycle is repeated (figure 2).

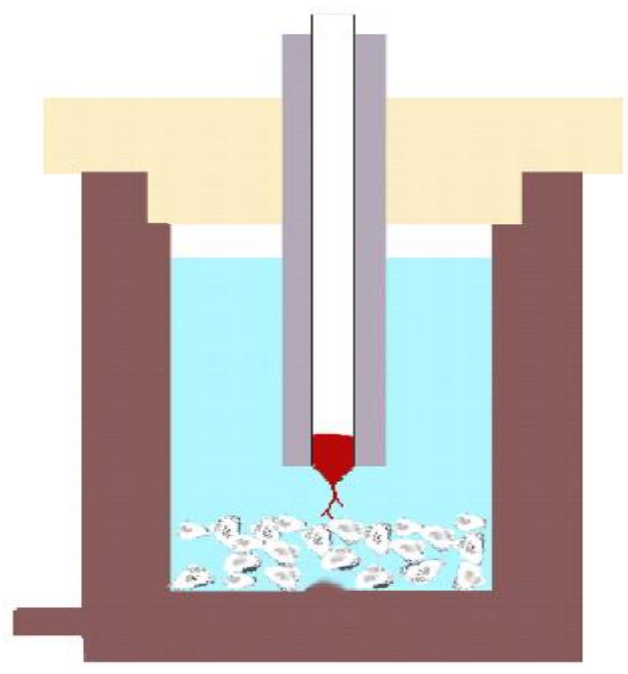

Fig.2. The process of crushing the mineral quartz. 
In experiments, the degree of quartz grinding was studied, which was determined by the number of particles of a certain diameter, where; $\mathrm{K}$ - the fraction of the specified value of the total volume, C-the capacity of the capacitor Bank, $\mathrm{d}_{0}, \mathrm{~d}$ - the diameters of the fractions of the initial and final product after electro-hydro-impulse processing (figure 3).

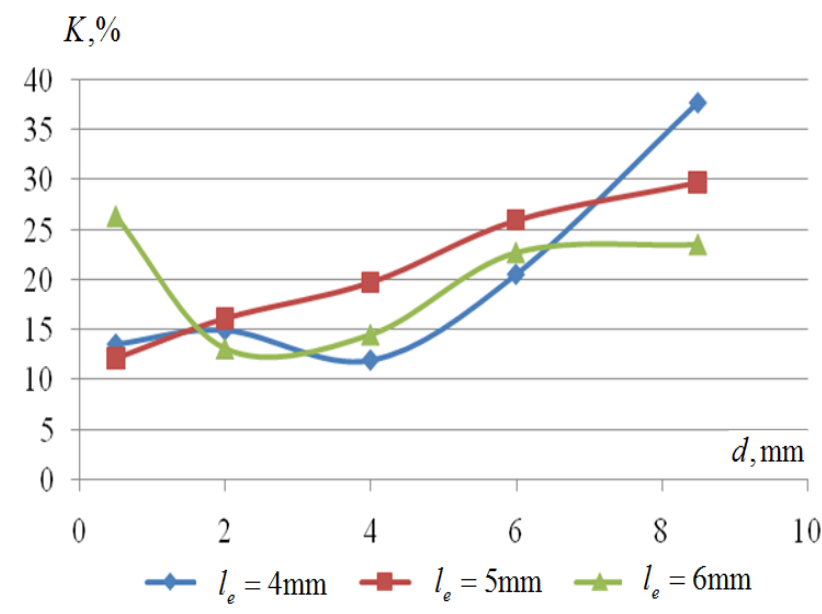

a)

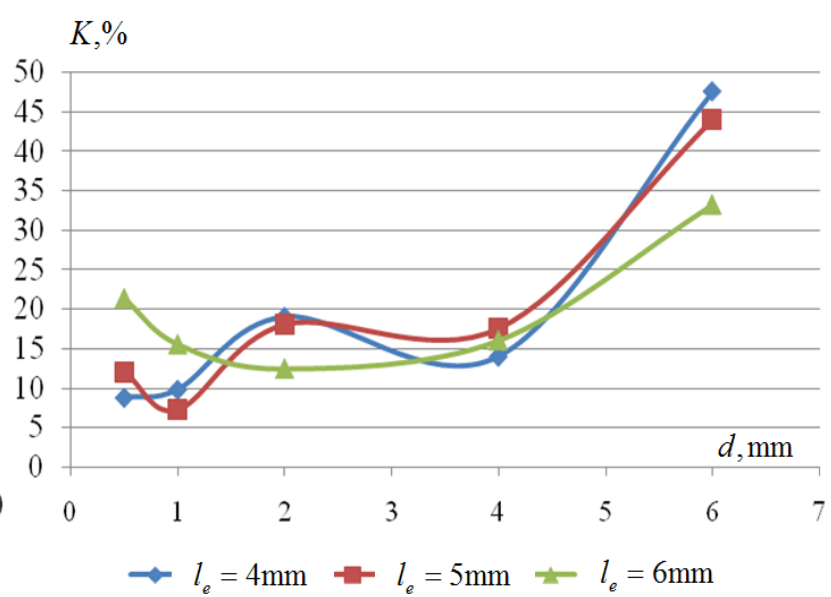

b)

Fig.3. Graphs of the dependence of the degree of quartz grinding on the diameter of the final product fractions: a) $\mathrm{C}=0.75 \mathrm{mkF}, \mathrm{d}_{0}=10 \mathrm{~mm}$; b) $\mathrm{C}=0.5 \mathrm{mkF}, \mathrm{d}_{0}=7 \mathrm{~mm}$.

In figure 3 , at the initial fraction with a diameter of $\mathrm{d}_{0}=10 \mathrm{~mm}$ and the length of the inter electrode distance of the working electrodes, the degree of grinding of small particles is $13.6 \%$, and at the degree of small particles increases to $37.8 \%$. When the diameter of the fractions $\mathrm{d}_{0}=7 \mathrm{~mm}$ and $\mathrm{C}=0.5 \mathrm{mkF}$ (figure $3, \mathrm{~b}$ ) we see that small particles with a diameter $\mathrm{d}>1 \mathrm{~mm}$ are crushed most intensively. At the length of the inter electrode distance of the working electrodes, the degree of grinding is $8.8 \%$, and at the degree of grinding was $21.3 \%$. The obtained experimental data show that when grinding the natural mineral quartz, the most optimal diameter of the fractions is $\mathrm{d} 0=7 \mathrm{~mm}$, and the optimal value of the capacitance of capacitor banks is $\mathrm{C}=0.5 \mathrm{mkF}$.

Figure 4 shows the results of tests performed at different discharge energy values on the $\mathrm{W}$ switching device.

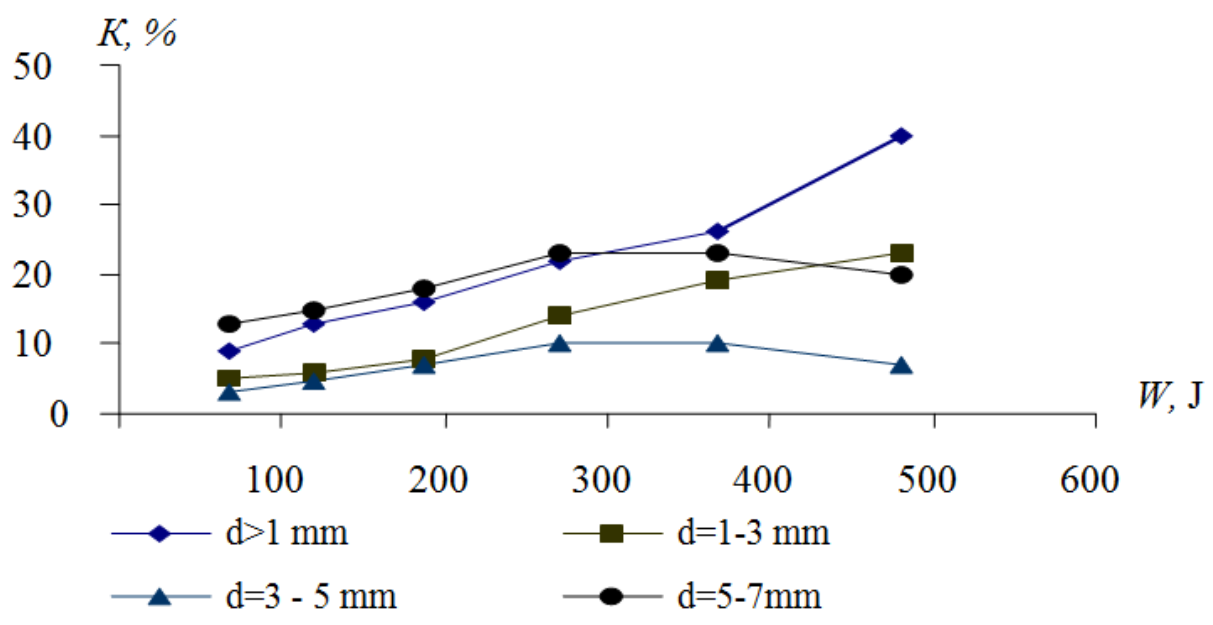

Fig.4. Graph of the dependence of the degree of quartz grinding on the discharge energy

$$
\left(\mathrm{C}=0.5 \mathrm{mkF}, \mathrm{d}_{0}=7 \mathrm{~mm}\right)
$$




\section{Conclusion}

Analysis of the research results allows us to conclude that the degree of grinding of the quartz mineral $(\mathrm{d}>1 \mathrm{~mm}$ and $\mathrm{d}=1-2 \mathrm{~mm})$ increases with increasing specific energy introduced into the discharge channel. The results obtained show that the spark gives energy through the surface of the shock wave propagation, therefore, the denser the material surrounds the discharge zone (the more crushed it is), the more rational and complete the energy allocated by the discharge channel is spent. In this regard, the electrohydraulic method of crushing into large fractions is less profitable compared to existing methods of crushing and, conversely, with an increase in the degree of grinding, electrohydraulic crushing becomes the most profitable method $[2,181 \mathrm{p}$.$] .$

The analysis of the research results allows us to conclude that the degree of grinding of the quartz mineral increases with the increase in the specific energy introduced into the discharge channel, which is explained by the fact that the processed ore first forms a network of micro cracks on the path of the shock wave, which creates a continuous stress state.

The developed method of electrohydraulic treatment of water suspensions of minerals allows quickly and with minimal costs to obtain a powder (mixture) with certain sizes of solid fractions, change its structure and characteristics, and on its basis to create new materials with specified properties, as well as simultaneously improve sanitary and hygienic working conditions and significantly reduce environmental pollution [7].

\section{REFERENCES}

1 Kulinich V.V., Sagunov V.G., Uzhkenov B.S. and others. Deposits of mining raw materials of Kazakhstan. Handbook. Almaty: 2000, Vol.1, 372 p. [in Russian]

2 Yutkin L.A. Electrohydraulic effect and its application in industry. Leningrad, Mashinostroenie, 1986, 253 p. [in Russian]

3 Semkin B. V., Usov A. F., Kurets V.I. Fundamentals of electrical impulse destruction of materials. Apatity: Publ. KNTS RAS, 1995, 189 p. [in Russian]

4 Kussaiynov K., Nussupbekov B.R., Bulkairova G.A., Omarova G.S. Electrohydroimpulse destruction of quartz mineral. Bulletin of the Karaganda University. Physics Series. 2004, No. 4 (36), pp. 73 -75 . [in Russian].

5 Bulkairova G.A., Shaiymerdenova G.M., Oktyabr A. Research microstructure and element composition of the mineral quartz deposit of Nadyrbay treat an electro-hydraulic method. Bulletin of the Karaganda University. Physics Series. 2015, No. 1 (77), pp. 66 - 71.

6 Kussaiynov K., Nussupbekov B.R. Shaimerdenova K.M., Bulkairova G. A., Khassenov A.K. Progressive methods of enrichment and complex processing of natural and technogenic mineral raw materials: Proceedings of the international meeting, Karaganda, 2015, pp. 129-132. [in Russian]

7 Bulkairova G.A., Nussupbekov B.R., Toktabolat G.G. Research of lectrohydraulic destruction of mineral quartz. Bulletin of the Karaganda University. Physics Series. 2018, No. 1 (89), pp. 81 - 87. 